\title{
Precise determination of the proton magnetic radius from electron scattering data
}

\author{
J. M. Alarcón $\odot,{ }^{1}$ D. W. Higinbotham $\odot,{ }^{2}$ and C. Weiss ${ }^{2}$ \\ ${ }^{1}$ Departamento de Física Teórica \& IPARCOS, Universidad Complutense de Madrid, 28040 Madrid, Spain \\ ${ }^{2}$ Jefferson Lab, Newport News, Virginia 23606, USA
}

(Received 25 February 2020; revised 10 August 2020; accepted 1 September 2020; published 21 September 2020)

\begin{abstract}
We extract the proton magnetic radius from high-precision electron-proton elastic scattering cross section data. Our theoretical framework combines dispersion analysis and chiral effective field theory and implements the dynamics governing the shape of the low- $Q^{2}$ form factors. It allows us to use data up to $Q^{2} \approx 0.5 \mathrm{GeV}^{2}$ for constraining the radii and overcomes the difficulties of empirical fits and $Q^{2} \rightarrow 0$ extrapolation. We obtain a magnetic radius $r_{M}^{p}=0.850 \pm 0.001$ ( $1 \sigma$ fit uncertainty) ${ }_{-0.004}^{+0.009}$ (full-range theory uncertainty) fm, significantly different from earlier results obtained from the same data using empirical fits, and close to our extracted electric radius $r_{E}^{p}=0.842 \pm 0.002$ ( $1 \sigma$ fit uncertainty) ${ }_{-0.002}^{+0.005}$ (full-range theory uncertainty) fm.
\end{abstract}

DOI: 10.1103/PhysRevC.102.035203

\section{INTRODUCTION}

The electromagnetic form factors (EM FFs) are the most basic expressions of the nucleon's finite spatial extent and composite internal structure. They describe the elastic response to external electric and magnetic fields as a function of the four-momentum transfer $Q^{2}$ and can be associated with the spatial distributions of charge and current in the nucleon. The traditional representation of FFs in terms of three-dimensional spatial densities at fixed instant time $x^{0}$ is appropriate only for nonrelativistic systems such as nuclei [1]. For relativistic systems such as hadrons, the spatial structure is expressed through two-dimensional transverse densities at fixed light-front time $x^{+}=x^{0}+x^{3}$. In the context of QCD these transverse densities can be regarded as projections of the nucleon's partonic structure (generalized parton distributions) [2-4]. The EM FFs thus reveal aspects of the spatial distribution of quarks and their orbital motion and spin and have become objects of great interest in nucleon structure studies $[5,6]$.

The values of the electric and magnetic proton FFs at $Q^{2}=0$ are given by the total charge and magnetic moment of the proton, $G_{E}^{p}(0)=1, G_{M}^{p}(0)=\mu^{p}=2.793$. The leading information about the spatial structure is in the first derivatives of the FFs at $Q^{2}=0$. They are conventionally expressed in terms of the equivalent electric and magnetic three-dimensional root-mean-square radii,

$$
\frac{d G_{E}^{p}}{d Q^{2}}(0)=-\frac{\left(r_{E}^{p}\right)^{2}}{6}, \quad \frac{1}{\mu^{p}} \frac{d G_{M}^{p}}{d Q^{2}}(0)=-\frac{\left(r_{M}^{p}\right)^{2}}{6} .
$$

Published by the American Physical Society under the terms of the Creative Commons Attribution 4.0 International license. Further distribution of this work must maintain attribution to the author(s) and the published article's title, journal citation, and DOI. Funded by $S C O A P^{3}$.
This does not imply an actual physical interpretation in terms of three-dimensional densities; the proper interpretation in terms of two-dimensional densities is discussed below [1]. Besides their importance for nucleon structure, the FF derivatives are needed in tests of atomic bound-state calculations in quantum electrodynamics and in precision measurements of the Rydberg constant $[7,8]$.

The proton electric (or charge) radius is extracted from the proton FFs measured in electron-proton elastic scattering and from the nuclear corrections to atomic energy levels of electronic and muonic hydrogen measured in precision spectroscopy experiments; see Refs. $[9,10]$ for a review. Apparent discrepancies between the different extraction methods (the "proton radius puzzle") have engendered intense experimental and theoretical efforts, including dedicated new elastic scattering experiments at low $Q^{2}$ with electron and muon beams [11,12]. Most recent experiments and reanalyses have converged around $r_{E}^{p}=0.84 \mathrm{fm}[11,13-24]$, while some have obtained larger values [25-28]; the CODATA Task Group and the Particle Data Group have now adopted $0.84 \mathrm{fm}$ as the recommended value $[29,30]$. The proton magnetic radius can only be extracted from elastic FF measurements (a method using atomic measurements was proposed in Ref. [31]). Empirical fits to the Mainz A1 data [32,33] have produced a range of values that disagree with each other, $r_{M}^{p}=0.78 \pm 0.02 \mathrm{fm}$ [32], $0.914 \pm 0.035 \mathrm{fm}$ and $0.776 \pm 0.038 \mathrm{fm}$ [25], and differ from the results of dispersive fits, $0.848_{-0.005}^{+0.006}$ ( $3 \sigma$ total uncertainty) fm [34], and other analyticity-based methods [35]. It is necessary to resolve these discrepancies and determine the proton magnetic radius with an overall accuracy and consistency comparable to those achieved in the electric radius.

Here we report an extraction of the proton magnetic radius from electron scattering data using a novel theoretical framework based on dispersion analysis and chiral effective field theory (DI $\chi$ EFT) $[20,36-38]$. It implements analyticity and the dynamics governing the shape of the low- $Q^{2}$ FFs and allows us to use data up to $Q^{2} \approx 0.5 \mathrm{GeV}^{2}$ for constraining the radii, increasing the sensitivity to the magnetic 
FF. It overcomes the difficulties in extraction methods based on empirical fits and $Q^{2} \rightarrow 0$ extrapolation (functional form bias, unstable extrapolation), particularly the issues related to the normalization of data sets taken at different incident energies. DI $\chi$ EFT was used in Ref. [20] to extract $r_{E}^{p}$ from an empirical FF parametrization [39] and delivered a value of $r_{E}^{p}=0.844 \pm 0.007 \mathrm{fm}$, in agreement with the other determinations quoted above and the recent results of the Jefferson Lab PRad experiment [11]. In this work we use the method to extract both $r_{M}^{p}$ and $r_{E}^{p}$ from a direct analysis of the cross section data, dominated by the Mainz A1 data [32,33]. We obtain $r_{M}^{p}=0.850 \pm 0.001$ ( $1 \sigma$ fit uncertainty) ${ }_{-0.004}^{+0.009}$ (full-range theory uncertainty) fm, significantly different from the values extracted from the same data using empirical fits [25,32], and surprisingly close to $r_{E}^{p}$. In the course we also update our previous extraction of $r_{E}^{p}$ and verify the robustness of the results.

\section{METHOD}

DI $\chi$ EFT is a method for calculating nucleon FFs combining dispersion analysis and chiral effective field theory. The theoretical foundations are described in detail in Refs. [36-38]; applications to FF fits are discussed in Ref. [20]. The FFs are represented as dispersion integrals over $t \equiv-Q^{2}$. The spectral functions on the two-pion cut at $t>4 M_{\pi}^{2}$ are calculated using (i) the elastic unitarity relation; (ii) $\pi N$ amplitudes computed in $\chi \mathrm{EFT}$ at leading order, next-to-leading order, and partial next-to-next-to-leading order accuracy; (iii) the timelike pion FF measured in $e^{+} e^{-}$ annihilation experiments. The approach includes $\pi \pi$ rescattering effects and the $\rho$ resonance and generates accurate spectral functions up to $t \approx 1 \mathrm{GeV}^{2}$. Higher-mass $t$-channel states are described by effective poles. The parameters specifying the dynamical input (the low-energy constants of the $\chi$ EFT calculation, and the strength of the effective poles) are fixed using the sum rules of dispersion theory and expressed in terms of the nucleon charges, magnetic moments, and radii. For each assumed value of $r_{E}^{p}$ and $r_{M}^{p}$ the theory generates a unique prediction for $G_{E}^{p}\left(Q^{2}\right)$ and $G_{M}^{p}\left(Q^{2}\right)$; the theoretical uncertainties of the predictions are discussed and estimated below [20]. The theory predicts the "shape" of the spacelike FF as determined by analyticity (position of singularities) and dynamics (strength of singularities). In this way the values of the radii are correlated with the predicted behavior of the FFs at finite momentum transfers $Q^{2} \approx 1 \mathrm{GeV}^{2}$, allowing the use of such data for radius extraction. A computer code generating the DI $\chi$ EFT FF predictions, example plots, and further information are available in the Supplemental Material [40].

For our radius extraction we use the high-precision data in electron-proton elastic scattering from the Mainz A1 experiment, which dominate the world data [32,33]. The experiment measured the elastic scattering cross section at momentum transfers $0.003 \lesssim Q^{2} \lesssim 1 \mathrm{GeV}^{2}$ and incident electron energies $E$ from 0.18 to $0.855 \mathrm{GeV}$ (see Fig. 1). In the one-photon exchange approximation the differential cross section is related to the proton electric and magnetic FFs by the standard
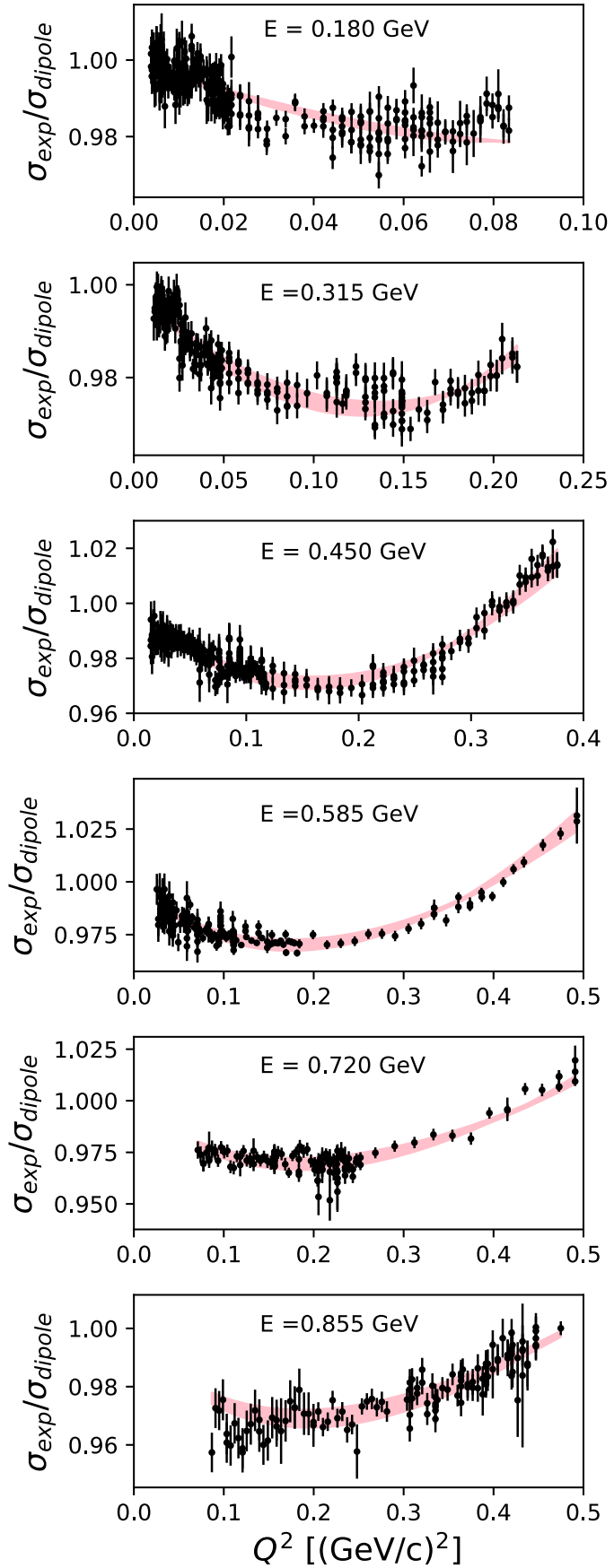

FIG. 1. Data: Mainz A1 electron-proton elastic scattering cross section data [32,33], with the normalization of the sets determined by our fit (in the higher energy bins, the plots show the data up to $Q^{2}=0.5 \mathrm{GeV}^{2}$ ). Bands: Theoretical model (DI $\left.\chi \mathrm{EFT}\right)$ with parameters $\left(r_{E}^{p}, r_{M}^{p}\right)$ obtained from our best fit. The bands show the range of the model predictions obtained by varying the parameters in the $1 \sigma$ confidence interval of the fit; it does not include the theoretical uncertainty of the model. Both data and model are divided by the cross section evaluated with the standard dipole FFs.

expression

$$
\frac{d \sigma}{d \Omega}=\left(\frac{d \sigma}{d \Omega}\right)_{\text {Mott }} \frac{\epsilon\left[G_{E}^{p}\left(Q^{2}\right)\right]^{2}+\tau\left[G_{M}^{p}\left(Q^{2}\right)\right]^{2}}{\epsilon(1+\tau)},
$$


where $(d \sigma / d \Omega)_{\text {Mott }}$ is the cross section for scattering from a spinless pointlike target,

$$
\epsilon=\frac{1-\frac{Q^{2}}{2 m E}\left(1+\frac{m}{2 E}\right)}{1-\frac{Q^{2}}{2 m E}\left(1-\frac{m}{2 E}\right)+\frac{1}{2}\left(\frac{Q^{2}}{2 m E}\right)^{2}}
$$

is the virtual photon polarization parameter (expressed here as a function of $Q^{2}$ and $E$ ), and $\tau=Q^{2} /\left(4 m^{2}\right)$, with $m$ the proton mass. For given $E$, the theoretical kinematic limits in $Q^{2}$ are

$$
0 \leqslant Q^{2} \leqslant \frac{2 m E}{1+\frac{m}{2 E}},
$$

corresponding to $1 \geqslant \epsilon \geqslant 0$. The expressions for $Q^{2}$ and $\epsilon$ in terms of the the scattering angle and the actual experimental limits of the variables in the A1 experiment are given in Ref. [33]. The relative contribution of the electric and magnetic FFs to the cross section is determined by the coefficients with which they enter in Eq. (2): $\left(G_{E}^{p}\right)^{2}$ enters with $\epsilon$ and contributes starting from $Q^{2}=0$, while $\left(G_{M}^{p}\right)^{2}$ enters with $\tau$ and contributes only at $Q^{2}>0$. The two-dimensional data cover the cross section at a given $Q^{2}$ at several values of $\epsilon$ and allow for separation of the contributions of $G_{E}^{p}$ and $G_{M}^{p}$ through global fits, generalizing the traditional Rosenbluth method [32,33].

An important issue in global fits is the normalization of the data sets taken at different energies. The normalization of the cross section data (both absolute and relative, between different energies) is limited by the knowledge of the absolute luminosity in the different settings and subject to considerable uncertainties. The combination of data taken at different energies therefore requires rescaling of the data sets, which depends on the functional form of the FFs or on other assumptions. In the context of empirical fits the effect of the rescaling on the random uncertainties of the data was studied in detail in Refs. [25,33]. In the context of our approach this problem is naturally solved by the fact that the theoretical model predicts the shape of the FFs at finite $Q^{2}$ (in dependence of the radii). We can therefore perform a global fit with floating normalizations of the data sets, which can adjust themselves to the theoretical model; the physical information is in the variation of the data with $Q^{2}$, which tests the theoretical predictions for the shape and fixes the radius parameters through the best fit.

As the figure of merit for the global fit with floating normalizations we use a $\chi^{2}$ function of the form

$$
\begin{gathered}
\chi^{2} \equiv \chi^{2}\left(r_{E}^{p}, r_{M}^{p} ; \Lambda_{1}, \ldots, \Lambda_{N_{\text {set }}}\right) \\
\equiv \sum_{i=1}^{N_{\text {dat }}}\left[\frac{\sigma_{\text {thy }, i}-\Lambda_{k(i)} \sigma_{\exp , i}}{\Lambda_{k(i)} \Delta \sigma_{\text {exp }, i}}\right]^{2}, \\
\sigma_{\text {thy }, i} \equiv \sigma\left(E_{i}, Q_{i}^{2}\right)\left[\mathrm{DI} \chi \text { EFT, params } r_{E}^{p}, r_{M}^{p}\right],
\end{gathered}
$$

where we use $\sigma \equiv d \sigma / d \Omega$ for brevity. The summation is over the $N_{\text {dat }}$ data points labeled by $i . \sigma_{\text {thy }, i}$ is the theoretical elastic scattering differential cross section, Eq. (2), at the kinematic point $\left(E_{i}, Q_{i}^{2}\right)$, evaluated with the DI $\chi$ EFT FFs $G_{E}^{p}$ and $G_{M}^{p}$ with the parameters $\left(r_{E}^{p}, r_{M}^{p}\right) . \sigma_{\exp , i}$ is the measured cross section and $\Delta \sigma_{\exp , i}$ is the random uncertainty. The data points are grouped in $N_{\text {set }}$ sets measured under the same running conditions; the normalization is assumed to be constant inside each set, but its value is unknown. The $N_{\text {set }}$ parameters $\left(\Lambda_{1}, \ldots, \Lambda_{N_{\text {set }}}\right)$ represent the floating normalizations in each set; $k(i)$ denotes the index $k$ of the set to which data point $i$ belongs. (A detailed discussion of how the experimental normalizations were defined and obtained can be found in Ref. [22]). The $\chi^{2}$ defined by Eq. (5) is thus a function of the theory parameters $\left(r_{E}^{p}, r_{M}^{p}\right)$ and the normalization parameters $\left(\Lambda_{1}, \ldots, \Lambda_{N_{\text {set }}}\right)$. Minimization is performed with respect to all the parameters simultaneously. The values of the $\Lambda_{k}(k=$ $\left.1, \ldots, N_{\text {set }}\right)$ at the minimum are found to be equal to unity within $\lesssim 1 \%$, indicating that the normalization determined in the original analysis of Ref. [33] is reproduced well by our fit; the values themselves have no physical significance otherwise (nuisance parameters). The values of $\left(r_{E}^{p}, r_{M}^{p}\right)$ at the minimum correspond to the best fit to the data and represent the proton radii extracted with our method.

To estimate the fit uncertainties of the extracted radii, we use the criterion $\Delta \chi^{2}=2.3$ to determine the $1 \sigma$ interval $(68 \%$ confidence interval), corresponding to the simultaneous estimation of two independent parameters. The uncertainties of the physical parameters $r_{E}^{p}$ and $r_{M}^{p}$ are affected also by the statistical fluctuations of the normalization parameters $\Lambda_{k}$; we have estimated the total statistical uncertainties using a bootstrap method and found them to be very close to the uncertainties of $r_{E}^{p}$ and $r_{M}^{p}$ one would obtain from the variation of $\chi^{2}$ in $r_{E}^{p}$ and $r_{M}^{p}$ after minimization with respect to $\left(\Lambda_{1}, \ldots, \Lambda_{N_{\mathrm{set}}}\right)$.

In our assessment of the uncertainties of the extracted radii we must include also the intrinsic theoretical uncertainty of the DI $\chi$ EFT model. This refers to the uncertainty in the model predictions for $G_{E}^{p}$ and $G_{M}^{p}$ for given values of $r_{E}^{p}$ and $r_{M}^{p}$, which results from the effective description of the high-mass states in the dispersion integral. For the electric FF this theoretical uncertainty was estimated in Ref. [20]; we now extend this estimate to the magnetic FF and the cross section predictions. The uncertainty is estimated by varying the position of the effective high-mass pole in the isovector electric and magnetic spectral functions over a range $M_{1}^{2}=(0.5-2) \times M_{1}^{2}(\mathrm{nom})$, where $M_{1}^{2}(\mathrm{nom}) \approx 2.1 \mathrm{GeV}^{2}$ is the nominal value determined in Ref. [38]. The values of $M_{1}^{2}$ covered in this way extend from the upper end of the two-pion continuum at $\approx 1 \mathrm{GeV}^{2}$ to the two-nucleon threshold at $4 m^{2}$ and represent the maximum plausible range of the effective pole position in the context of this model [20]. The theoretical uncertainty of the cross section predictions obtained in this way is shown in Fig. 2 for several (assumed) values of $r_{M}^{p}$. The figure thus simultaneously illustrates the sensitivity of the DI $\chi$ EFT cross section prediction to the magnetic radius and the theoretical uncertainty associated with the prediction for each radius, as well as the dependence of either on $E$ and $Q^{2}$. One observes the following: (a) For all values of $E$ shown here, in the region $Q^{2} \lesssim 0.1 \mathrm{GeV}^{2}$ the theoretical uncertainty of the cross section predictions is small, but there is little sensitivity to the magnetic radius, because the magnetic FF enters in the cross section with a coefficient $\propto Q^{2}$; cf., Eq. (2). (b) For $E=0.450 \mathrm{GeV}$ and $Q^{2} \approx 0.1-0.4 \mathrm{GeV}^{2}$ the theoretical uncertainty of the cross section predictions is 

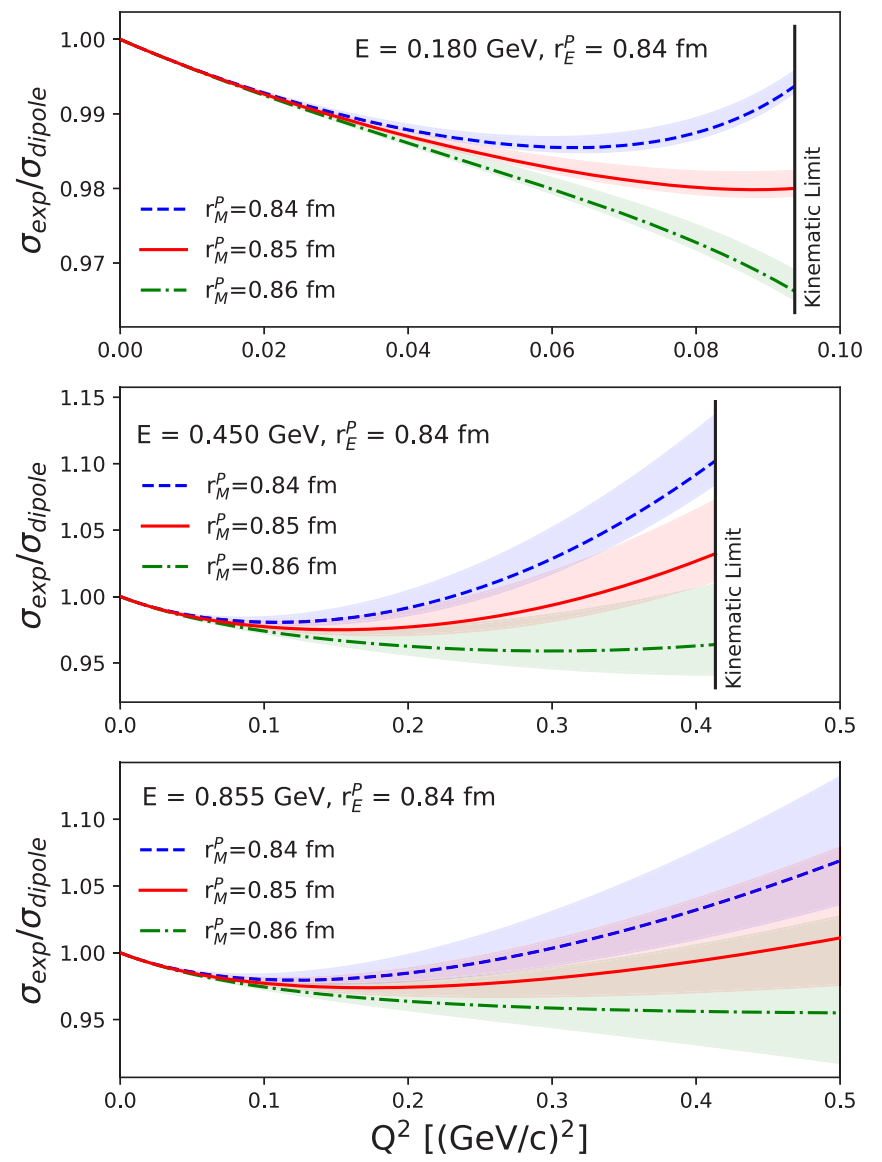

FIG. 2. Lines: DI $\chi$ EFT model predictions for the electronproton elastic scattering cross section obtained with magnetic radii $r_{M}^{p}=0.84 \mathrm{fm}$ (dashed lines), $0.85 \mathrm{fm}$ (solid lines), and $0.86 \mathrm{fm}$ (dashed-dotted lines); the electric radius $r_{E}^{p}$ is kept constant at $0.84 \mathrm{fm}$. Associated shaded bands: Theoretical uncertainty of the $\mathrm{DI} \chi \mathrm{EFT}$ predictions for a given radius, resulting from the parametrization of the high-mass states in the dispersion integral (see text). The three plots show the cross section as a function of $Q^{2}$, at $E=0.180,0.450$, and $0.855 \mathrm{GeV}$. In the first two plots, the kinematic upper limit of $Q^{2}$ is marked by vertical lines; in the third plot it is outside the plotted region. The model cross section is divided by the one evaluated with the standard dipole FFs (cf., Fig. 1).

moderate, while at the same time there is good sensitivity to the magnetic radius. This region of $E$ and $Q^{2}$ has the most impact on our magnetic radius extraction (see below). (c) For $E=0.855 \mathrm{GeV}$ and $Q^{2} \approx 0.1-0.5 \mathrm{GeV}^{2}$, the ratio of theoretical uncertainties to magnetic radius sensitivity is less favorable than at $E=0.450 \mathrm{GeV}$ because $\epsilon$ is larger [see Eq. (3)], and the electric FF is more dominant (the electric FF contributes to the theoretical uncertainty of the cross section prediction but not to the magnetic radius sensitivity).

To quantify the effect of the theoretical uncertainty on the radius extraction, we perform the fits and $\chi^{2}$ minimization with the nominal and the changed values of $M_{1}^{2}$ and take the differences between the results as a measure of the uncertainty. We regard this uncertainty as the "full plausible range" of the theoretical model and quote it separately from the statistical uncertainty obtained from the fit.
The kinematic range of the fit used for our radius extraction is determined by a balance of several considerations: (a) the sensitivity of the cross section to the magnetic radius, which requires $Q^{2} \gtrsim 0.1 \mathrm{GeV}^{2}$; (b) the experimental uncertainties of the data at different $E$ and $Q^{2}$, which control their impact on the fit; and (c) the theoretical uncertainties of the DI $\chi$ EFT model. For the electric radius extraction from the electric FF these considerations were described in detail in Ref. [20]; we now extend them to include the magnetic FF and performing the analysis at the cross section level. We include in the fit the cross section data from all available $E$ sets up to a fixed maximum momentum transfer, $Q^{2}<Q_{\max }^{2}$; in the sets where the experimental upper limit of $Q^{2}$ is smaller than $Q_{\max }^{2}$ we take all the data up to the limit. Our standard value is $Q_{\max }^{2}=$ $0.5 \mathrm{GeV}^{2}$; this choice ensures good sensitivity to the magnetic radius, while the theoretical uncertainties are still moderate (see Fig. 2). We test the robustness of the radius extraction by performing fits with different values of $Q_{\max }^{2}$, including fewer or more data in the fit.

\section{RESULTS}

Our standard fit with $Q_{\max }^{2}=0.5 \mathrm{GeV}^{2}$ includes 1285 of the 1422 Mainz A1 data points. The overall quality of the description of the experimental cross sections is shown in Fig. 1. One observes the following: (a) The kinematic dependencies of the data (with the floating normalization determined by the fit) are well reproduced by the theoretical model. The statistically significant deviations observed in some $E$ and $Q^{2}$ regions are likely due to systematic effects in the data (some of these features cannot be reproduced even with empirical fits; cf. the discussion in Ref. [22]). (b) Most of the impact on the fit comes from data in the midrange $E$ bins $(0.45,0.585$, $0.72 \mathrm{GeV}$ ), where the data are most precise.

The reduced $\chi^{2}$ profile in the physical parameters $r_{E}^{p}$ and $r_{M}^{p}$, obtained after minimization in the normalization parameters, is shown in Fig. 3; the minimization in the normalization parameters has been performed separately for each given value of $r_{E}^{p}$ and $r_{M}^{p}$. One observes that (a) the variations of $\chi^{2}$ in $r_{E}^{p}$ and $r_{M}^{p}$ are approximately independent and (b) clear minima are obtained in both parameters. Minimizing with respect to the radii, we extract $r_{E}^{p}=0.842 \pm 0.002 \mathrm{fm}$ and $r_{M}^{p}=0.850 \pm 0.001 \mathrm{fm}$ with a reduced $\chi^{2}$ of 1.39 . (The interpretation of the $\chi^{2}$ value and its significance for the radius extraction are investigated below).

To test the robustness of the results, we have performed fits with different values of $Q_{\max }^{2}$ and found little effect on the extracted radii. In fact, using the entire Mainz A1 data set up to $Q_{\max }^{2}=1 \mathrm{GeV}^{2}$ gives $r_{E}^{p}=0.843 \pm 0.002 \mathrm{fm}$ and $r_{M}^{p}=0.850 \pm 0.001 \mathrm{fm}$ with a reduced $\chi^{2}$ of 1.43 . This shows that the theoretical model (evaluated at the "best" value of the radii) accurately describes the $Q^{2}$ dependence of the data over the entire range considered.

The minimum $\chi^{2}$ values obtained in our fits (after minimization with respect to the normalization parameters and the radii) depend on the uncertainties assigned to the experimental data and need to be interpreted as such. The original analysis of the A1 data of Ref. [33] made certain assumptions about 


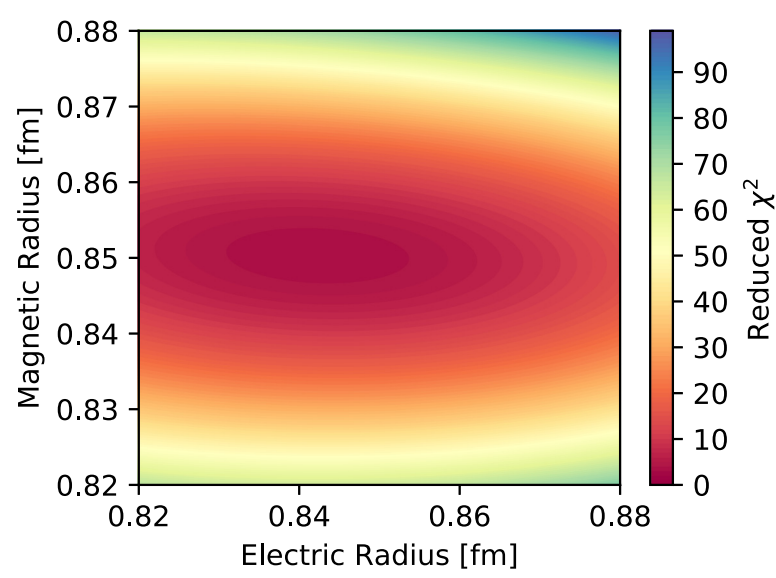

FIG. 3. The reduced $\chi^{2}$ obtained from Eq. (5), after minimization in the normalization parameters, as a function of $r_{E}^{p}$ and $r_{M}^{p}$. The minimization in the normalization parameters has been performed separately for each given value of $r_{E}^{p}$ and $r_{M}^{p}$.

the probability distribution of the errors in estimating the uncertainties. The reanalysis of Ref. [25] reevaluated these assumptions and used the actual distributions of the data (from repeated measurements at the same kinematic points) to determine the uncertainties, obtaining slightly larger uncertainties than Ref. [33]. To investigate the impact on our radius extraction, we have performed fits both to the reanalyzed data and uncertainties of Ref. [25] and the original ones of Ref. [33] and compared the results. The fit with $Q_{\max }^{2}=0.5 \mathrm{GeV}^{2}$ uses 569 of the 658 reanalyzed data points (the reanalysis combines the data points from repeated measurements) and gives radii $r_{E}^{p}=0.840 \pm 0.002 \mathrm{fm}$ and $r_{M}^{p}=0.849 \pm 0.001 \mathrm{fm}$ with a reduced $\chi^{2}$ of 1.07 , in good agreement with our fit to the original data. Extending $Q_{\max }^{2}$ to include all the reanalyzed data we obtain $r_{E}^{p}=0.841 \pm 0.003 \mathrm{fm}$ and $r_{M}^{p}=0.849 \pm 0.001$ fm with a reduced $\chi^{2}$ of 1.10 , showing similar stability as the fit to the original data. One sees that the fits to the reanalyzed data have significantly lower $\chi^{2}$ than those to the original data, while the extracted radii and their uncertainties are essentially the same. This shows that the larger $\chi^{2}$ values obtained in the fits to the original data only reflect the underestimated uncertainties of the original data but have no effect on the extracted radii and their uncertainties. Altogether, our tests show that the extracted radii are not sensitive to the choice of data sets used in the fits.

We have estimated the theoretical uncertainty of the radius extraction by performing the fits with the changed values of the high-mass pole position as described in Sec. II. The "full plausible range" theoretical uncertainties obtained in this way are $r_{E}^{p}=0.842_{-0.002}^{+0.005} \mathrm{fm}$ and $r_{M}^{p}=0.850_{-0.004}^{+0.009} \mathrm{fm}$. We note that the reduced $\chi^{2}$ of the fit (after minimizing with the respect to the radii) changes only by $<12 \%$ when varying $M_{1}^{2}$ over the entire range $(0.5-2) \times M_{1}^{2}$ (nom). This clearly shows the different role of the parameters in our DI $\chi$ EFT-based radius extraction: the radii $r_{E}^{p}$ and $r_{M}^{p}$ are "fit parameters" to be determined by $\chi^{2}$ variation; the pole mass $M_{1}^{2}$ is a "theory parameter" to be varied in a plausible range, with essentially no impact from the data in the range considered here.
Summarizing the above results of the fits, tests, and uncertainty estimates, our final results for the extracted electric and magnetic radii are

$$
\begin{aligned}
r_{E}^{p}= & 0.842 \pm 0.002 \text { (1 } \sigma \text { fit uncertainty) } \\
& { }_{-0.002}^{+0.005} \text { (full-range theory uncertainty) } \mathrm{fm}, \\
r_{M}^{p}= & 0.850 \pm 0.001(1 \sigma \text { fit uncertainty) } \\
& { }_{-0.004}^{+0.009} \text { (full-range theory uncertainty) fm. }
\end{aligned}
$$

One observes that the fit uncertainty is smaller in $r_{M}^{p}$ than in $r_{E}^{p}$ (see also Fig. 3), while the theory uncertainty is larger in $r_{M}^{p}$ than in $r_{E}^{p}$. This pattern is explained by the different sensitivity of the cross section to $G_{M}^{p}$ and $G_{E}^{p}$; see Eq. (2) and the discussion in Sec. II. The sensitivity to $G_{M}^{p}$ is in the region of "higher" $Q^{2} \gtrsim 0.1 \mathrm{GeV}^{2}$, where the data are relatively more precise and have more constraining power in the fit (see Fig. 1), while at the same time the theoretical uncertainties are relatively larger (see Fig. 2). The sensitivity to $G_{M}^{p}$ is distributed more broadly and includes also "lower" $Q^{2} \lesssim 0.1 \mathrm{GeV}^{2}$, where the data are less precise but theoretical errors are small.

\section{DISCUSSION}

Several aspects of our method and results merit further discussion. Our theory-based method allows us to determine the proton's magnetic radius with a precision comparable to the electric one. This is because the dispersion-theoretical framework correlates the values of the radii with the behavior of the FFs at finite $Q^{2} \approx 0.1-0.5 \mathrm{GeV}^{2}$, where the magnetic FF contributes to the cross section with a strength comparable to the electric one. It is different from empirical fits such as higher-order polynomial fits, where the accurate extrapolation of the functions to $Q^{2} \rightarrow 0$ conflicts with the vanishing sensitivity of the cross section to the magnetic FF. Our method therefore offers principal advantages for the analysis of the proton's magnetic structure.

The results of our analysis validate previous results for the proton magnetic radius $\approx 0.85 \mathrm{fm}$, obtained using dispersive fits of the Mainz A1 [34,41] and earlier world data [42] (see references therein for earlier works). They disagree with the results obtained from various empirical fits of the Mainz A1 data [25,32]. This indicates that the observed discrepancies are due to the extraction methods (analyticity, correlations between $Q^{2}$ regions from dispersion relations) rather than the different data sets.

The Jefferson Lab PRad experiment has reported a new measurement of the electron-proton elastic cross section down to $Q^{2} \approx 10^{-4} \mathrm{GeV}^{2}$, significantly extending the reach of earlier measurements [11]. We have performed a fit including the PRad data in addition to the Mainz A1 data and found no change in the extracted $r_{E}^{p}$ and $r_{M}^{p}$ within uncertainties. This happens because the DI $\chi$ EFT model naturally describes the $Q^{2}$ dependence of the low- $Q^{2}$ data, with the same value of $r_{E}^{p}$ as favored by the higher- $Q^{2}$ data [20]; this was also observed in the analysis of Ref. [23]. Note that the low $-Q^{2}$ data are sensitive mostly to $r_{E}^{p}$, and that our present extraction of $r_{M}^{p}$ requires us to include data up to $Q^{2} \approx 0.5 \mathrm{GeV}^{2}$. 
The values of the electric and magnetic radii extracted from the data are very close. While this may be accidental, it is qualitatively consistent with the nonrelativistic quark model picture (independent particle motion in an $L=0$ orbital, no spin-orbit interactions). Using our method we can also determine the proton's transverse charge and magnetization radii, which refer to the relativistic representation of the FFs in terms of transverse densities and can be related to the generalized parton distributions [1-4]. The derivatives at $Q^{2}=0$ of the Dirac and Pauli FFs, $F_{1}^{p}$ and $F_{2}^{p}$, are related to those of the electric and magnetic FFs by

$$
\begin{gathered}
\frac{d F_{1}^{p}}{d Q^{2}}(0)=\frac{d G_{E}^{p}}{d Q^{2}}(0)+\frac{\kappa^{p}}{4 m^{2}}, \\
\frac{1}{\kappa^{p}} \frac{d F_{2}^{p}}{d Q^{2}}(0)=\frac{1}{\kappa^{p}}\left[\frac{d G_{M}^{p}}{d Q^{2}}(0)-\frac{d G_{E}^{p}}{d Q^{2}}(0)\right]-\frac{1}{4 m^{2}},
\end{gathered}
$$

where $\kappa^{p} \equiv F_{2}^{p}(0)=\mu^{p}-1$ is the proton anomalous magnetic moment. In the transverse density representation these derivatives determine the mean squared transverse radii of the distributions of charge and magnetization in the proton [1],

$$
-4 \frac{d F_{1}^{p}}{d Q^{2}}(0)=\left\langle b^{2}\right\rangle_{1}^{p}, \quad-\frac{4}{\kappa^{p}} \frac{d F_{2}^{p}}{d Q^{2}}(0)=\left\langle b^{2}\right\rangle_{2}^{p} .
$$

Equations (1) and (9)-(11) linearly relate $\left\langle b^{2}\right\rangle_{1}^{p}$ and $\left\langle b^{2}\right\rangle_{2}^{p}$ to $\left(r_{E}^{p}\right)^{2}$ and $\left(r_{M}^{p}\right)^{2}$. Using the results of our fit, we obtain $\left\langle b^{2}\right\rangle_{1}=0.394 \pm 0.002$ ( $1 \sigma$ fit uncertainty) ${ }_{-0.002}^{+0.005}$ (full-range theory uncertainty) $\mathrm{fm}^{2}$ and $\left\langle b^{2}\right\rangle_{2}=0.531 \pm 0.002$ ( $1 \sigma$ fit uncertainty) ${ }_{-0.006}^{+0.013}$ (full-range theory uncertainty) $\mathrm{fm}^{2}$. It is interesting to note that, if one neglected the small difference between the extracted electric and magnetic radii and set $\left(r_{E}^{p}\right)^{2}=\left(r_{M}^{p}\right)^{2}$, the transverse charge and magnetization radii would be related as

$$
\left\langle b^{2}\right\rangle_{2}^{p}-\left\langle b^{2}\right\rangle_{1}^{p}=\mu^{p} / m^{2} \quad \text { if } \quad\left(r_{E}^{p}\right)^{2}=\left(r_{M}^{p}\right)^{2},
$$

i.e., the difference would be entirely proportional to the proton magnetic moment. Equation (12) is the partonic expression of the approximate equality of the electric and magnetic radii.

\section{ACKNOWLEDGMENTS}

This material is based upon work supported by the U.S. Department of Energy, Office of Science, Office of $\mathrm{Nu}$ clear Physics under Contract No. DE-AC05-06OR23177. J.M.A. acknowledges support from the Community of Madrid through the Programa de atracción de talento investigador 2017 (Modalidad 1), and the Spanish MECD grants FPA201677313-P.
[1] G. A. Miller, Phys. Rev. C 99, 035202 (2019).

[2] M. Diehl, Phys. Rep. 388, 41 (2003).

[3] A. V. Belitsky and A. V. Radyushkin, Phys. Rep. 418, 1 (2005).

[4] S. Boffi and B. Pasquini, Riv. Nuovo Cim. 30, 387 (2007).

[5] V. Punjabi, C. F. Perdrisat, M. K. Jones, E. J. Brash, and C. E. Carlson, Eur. Phys. J. A 51, 79 (2015).

[6] S. Pacetti, R. Baldini Ferroli, and E. Tomasi-Gustafsson, Phys. Rep. 550-551, 1 (2015).

[7] M. I. Eides, H. Grotch, and V. A. Shelyuto, Phys. Rep. 342, 63 (2001).

[8] R. Pohl et al., Nature (London) 466, 213 (2010).

[9] R. Pohl, R. Gilman, G. A. Miller, and K. Pachucki, Annu. Rev. Nucl. Part. Sci. 63, 175 (2013).

[10] C. E. Carlson, Prog. Part. Nucl. Phys. 82, 59 (2015).

[11] W. Xiong et al., Nature (London) 575, 147 (2019).

[12] R. Gilman et al. (MUSE Collaboration), arXiv:1709.09753.

[13] M. Horbatsch and E. A. Hessels, Phys. Rev. C 93, 015204 (2016).

[14] K. Griffioen, C. Carlson, and S. Maddox, Phys. Rev. C 93, 065207 (2016).

[15] D. W. Higinbotham, A. A. Kabir, V. Lin, D. Meekins, B. Norum, and B. Sawatzky, Phys. Rev. C 93, 055207 (2016).

[16] M. Horbatsch, E. A. Hessels, and A. Pineda, Phys. Rev. C 95, 035203 (2017).

[17] A. Beyer et al., Science 358, 79 (2017).

[18] T. B. Hayward and K. A. Griffioen, Nucl. Phys. A 999, 121767 (2020).

[19] S. Zhou, P. Giuliani, J. Piekarewicz, A. Bhattacharya, and D. Pati, Phys. Rev. C 99, 055202 (2019).
[20] J. M. Alarcón, D. W. Higinbotham, C. Weiss, and Z. Ye, Phys. Rev. C 99, 044303 (2019).

[21] N. Bezginov, T. Valdez, M. Horbatsch, A. Marsman, A. C. Vutha, and E. A. Hessels, Science 365, 1007 (2019).

[22] S. K. Barcus, D. W. Higinbotham, and R. E. McClellan, Phys. Rev. C 102, 015205 (2020).

[23] M. Horbatsch, Phys. Lett. B 804, 135373 (2020).

[24] M. Mihovilovič, D. W. Higinbotham, M. Bevc, and S. Širca, Front. Phys. 8, 36 (2020).

[25] G. Lee, J. R. Arrington, and R. J. Hill, Phys. Rev. D 92, 013013 (2015).

[26] J. Arrington and I. Sick, J. Phys. Chem. Ref. Data 44, 031204 (2015).

[27] I. Sick and D. Trautmann, Phys. Rev. C 95, 012501(R) (2017).

[28] H. Fleurbaey, S. Galtier, S. Thomas, M. Bonnaud, L. Julien, F. Biraben, F. Nez, M. Abgrall, and J. Guéna, Phys. Rev. Lett. 120, 183001 (2018).

[29] E. Tietsinga et al. (CODATA Task Group on Fundamental Constants), 2018 adjustment of fundamental physics constants, available at https://www.nist.gov/pml/fundamental-physicalconstants.

[30] P. A. Zyla et al. (Particle Data Group), Prog. Theor. Exp. Phys. 2020, 083 C01 (2020).

[31] S. G. Karshenboim, Phys. Rev. D 90, 053013 (2014).

[32] J. C. Bernauer et al. (A1 Collaboration), Phys. Rev. Lett. 105, 242001 (2010).

[33] J. C. Bernauer et al. (A1 Collaboration), Phys. Rev. C 90, 015206 (2014). 
[34] I. T. Lorenz, Ulf-G. Meißner, H.-W. Hammer, and Y. B. Dong, Phys. Rev. D 91, 014023 (2015).

[35] Z. Epstein, G. Paz, and J. Roy, Phys. Rev. D 90, 074027 (2014).

[36] J. M. Alarcón and C. Weiss, Phys. Rev. C 96, 055206 (2017).

[37] J. M. Alarcón and C. Weiss, Phys. Rev. C 97, 055203 (2018).

[38] J. M. Alarcón and C. Weiss, Phys. Lett. B 784, 373 (2018).

[39] Z. Ye, J. Arrington, R. J. Hill, and G. Lee, Phys. Lett. B 777, 8 (2018).
[40] See Supplemental Material at http://link.aps.org/supplemental/ 10.1103/PhysRevC.102.035203 for a Jupyter Notebook generating the DI $\chi$ EFT FF predictions [20] and preparing reference plots.

[41] I. Lorenz, H.-W. Hammer, and U.-G. Meissner, Eur. Phys. J. A 48, 151 (2012).

[42] M. A. Belushkin, H.-W. Hammer, and Ulf-G. Meißner, Phys. Rev. C 75, 035202 (2007). 